\title{
The transient variation in the complexes of the low-latitude ionosphere within the equatorial ionization anomaly region of Nigeria
}

\author{
A. B. Rabiu ${ }^{1,2}$, B. O. Ogunsua ${ }^{1}$, I. A. Fuwape ${ }^{1}$, and J. A. Laoye ${ }^{3}$ \\ ${ }^{1}$ Space Physics Laboratory, Department of Physics, Federal University of Technology, Akure, Nigeria \\ ${ }^{2}$ Centre for Atmospheric Research, National Space Research and Development Agency, Anyigba, Nigeria \\ ${ }^{3}$ Department of Physics, Olabisi Onabanjo University, Ago-Iwoye, Nigeria
}

Correspondence to: B. O. Ogunsua (iobogunsua@futa.edu.ng)

Received: 9 November 2014 - Published in Nonlin. Processes Geophys. Discuss.: 5 December 2014

Revised: 23 August 2015 - Accepted: 25 August 2015 - Published: 11 September 2015

\begin{abstract}
The quest to find an index for proper characterization and description of the dynamical response of the ionosphere to external influences and its various internal irregularities has led to the study of the day-to-day variations of the chaoticity and dynamical complexity of the ionosphere. This study was conducted using Global Positioning System (GPS) total electron content (TEC) time series, measured in the year 2011, from five GPS receiver stations in Nigeria, which lies within the equatorial ionization anomaly region. The non-linear aspects of the TEC time series were obtained by detrending the data. The detrended TEC time series were subjected to various analyses to obtain the phase space reconstruction and to compute the chaotic quantifiers, which are Lyapunov exponents LE, correlation dimension, and Tsallis entropy, for the study of dynamical complexity. Considering all the days of the year, the daily/transient variations show no definite pattern for each month, but day-to-day values of Lyapunov exponents for the entire year show a wavelike semiannual variation pattern with lower values around March, April, September and October. This can be seen from the correlation dimension with values between 2.7 and 3.2, with lower values occurring mostly during storm periods, demonstrating a phase transition from higher dimension during the quiet periods to lower dimension during storms for most of the stations. The values of Tsallis entropy show a similar variation pattern to that of the Lyapunov exponent, with both quantifiers correlating within the range of 0.79 to 0.82 . These results show that both quantifiers can be further used together as indices in the study of the variations of the dynamical com-
\end{abstract}

plexity of the ionosphere. The presence of chaos and high variations in the dynamical complexity, even in quiet periods in the ionosphere, may be due to the internal dynamics and inherent irregularities of the ionosphere which exhibit non-linear properties. However, this inherent dynamics may be complicated by external factors like geomagnetic storms. This may be the main reason for the drop in the values of the Lyapunov exponent and Tsallis entropy during storms. The dynamical behaviour of the ionosphere throughout the year, as described by these quantifiers, was discussed in this work.

\section{Introduction}

The behaviour of natural systems like the ionosphere is a function of changes that occur in the underlying dynamics that exists in such a system. These underlying dynamics however can sometimes be complex and non-linear due to superposition of different changes in the dynamical variables that constitute it. However, there is no totally deterministic system in nature, because all natural systems exhibit a mixture of both stochastic and deterministic properties. Although few natural systems have been found to be low-dimensional deterministic in the sense of the theory, the concept of lowdimensional chaos has been proven to be fruitful in the understanding of many complex phenomena (Hegger et al., 1999). The degree of determinism or stochasticity in most natural systems is dependent on how much the system can be influenced by external factors, the nature of these external factors 
etc. The ionosphere, like every other natural system, possesses its intrinsic dynamics, and it can also be influenced by other external factors. The typical characteristics of a dynamical system like the ionosphere are expected to naturally show the interplay between determinism and stochasticity simply because of the fact that the ionosphere, which has an inherent internal dynamics, is also influenced by the influx of stochastic drivers like the solar wind, since it is influenced by external dynamics like every other natural system. This has made pure determinism impossible in the ionosphere, a situation that is common to every natural system and its surroundings.

The intensity of the solar wind coming into the ionosphere varies with the solar activity and this can sometimes result in geomagnetic storms and substorms driven in highintensity plasma wind at enormous speed, and it serves as a major stochastic driver leading to storms. The solar wind is driven from the Sun into the ionospheric system during the quiet and storm and during relatively quiet periods of each month of the year. However, other processes include various factors like local time variations of the neutral winds, ionization processes, production-recombination rates, photoionization processes, plasma diffusion and various electrodynamics processes (Unnikrishnan, 2010). The mesosphere and the lower thermospheric dynamics as reported by Kazimirovsky and Vergasova (2009) and the influence of gravity waves as reported by Sindelarova (2009) can also be of great influence on the internal dynamics of the ionosphere.

Therefore, it is of great importance to study the chaoticity and dynamical complexity of the ionosphere and its variations in all geophysical conditions. However, a good number of investigations have been carried out on the concept of chaos in the upper atmosphere before now, which includes the study on magnetospheric dynamics and the ionosphere. The study of chaos in magnetospheric index time series such as $\mathrm{AE}$ and $\mathrm{AL}$ were initially carried out by Vassiliadis et al. (1990), Shan et al. (1999) and Pavlos et al. (1992). These previous efforts made by the aforementioned researchers has led to the development of the concept of investigating and revealing the chaoticity and the complex dynamics of the ionosphere, and as a result, studies on the chaoticity of the ionosphere have been conducted, by some investigators like Bhattacharyya (1990), who studied chaotic behaviour of ionospheric diversity fluctuation using amplitude and phase scintillation data, and found the existence of low-dimension chaos. Also, Wernik and Yeh (1994) further revealed the chaotic behaviour of the ionospheric turbulence using scintillation data and numerical modelling of scintillation at high latitude. They showed that the ionospheric turbulence attractor (if it exists) cannot be reconstructed from amplitude scintillation data, and their measured phase scintillation data adequately reproduce the assumed chaotic structure in the ionosphere. Also, Kumar et al. (2007) reported the evidence of chaos in the ionosphere by showing the chaotic nature of the underlying dynamics of the fluctuations of the total electron content (TEC) power spectrum, indicating exponential decay and the calculated positive value of the Lyapunov exponent. This is also supported by the results of the comparison of the chaotic characteristics of the time series of variations of TEC with the pseudochaotic characteristic of the coloured noise time series. Xuann et al. (2006) studied chaos properties of ionospheric TEC using TEC data from 1996 to 2004, and analyse the possibility of predicting it by using chaos. They found the presence of chaos in the TEC measured in the study area, as indicated by the positive Lyapunov exponent computed from their data. The correlation dimension was 3.6092 from their estimation. They were also able to show that the TEC time series can be predicted using chaos.

Also, Unnikrishnan et al. (2006a, b) have analysed the deterministic chaos at mid-latitudes and Unnikrishnan (2010) and Unnikrishnan and Ravindran (2010) analysed some TEC data from some Indian low-latitude stations for quiet periods and major storm periods, and found in their results the presence of chaos, which was indicated by a positive Lyapunov exponent, and they also inferred that storm periods exhibit lower values compared to quiet periods. The dynamical complexity of magnetospheric processes and the ionosphere have been studied by a number of researchers. Balasis et al. (2008), Kazimirovsky et al. (2006) and Kazimirovsky and Vergasova (2009) investigated the dynamical complexity of the magnetosphere by using Tsallis entropy as a dynamical complexity measure in $D_{\text {st }}$ time series; Balasis et al. (2009) also investigated the dynamical complexity in $D_{\text {st }}$ further by considering different entropy measures. Coco et al. (2011), using the information theory approach, studied the dynamical changes of the polar cap potential which is characteristic of the polar region ionosphere by considering three cases: (i) steady interplanetary magnetic field (IMF) $B_{z}>0$, (ii) steady IMF $B_{z}<0$ and (iii) a double rotation from negative to positive and then positive to negative $B_{z}$. They observed a neat dynamical topological transition when the IMF $B_{z}$ turns from negative to positive and vice versa, pointing toward the possible occurrence of an order-disorder phase transition, which is the counterpart of the large-scale convection rearrangement and of the increase of the global coherence. Further studies on the chaotic behaviour and non-linear dynamics of the ionosphere over the low-latitude African region is however required for the improvement of our understanding and characterization of its dynamical behaviour. Recently, Ogunsua et al. (2014) studied comparatively the chaoticity of the equatorial ionosphere over Nigeria using TEC data, considering five quietest day classifications and five most disturbed day classifications. They were able to show the presence of chaos as indicated the positive Lyapunov exponents and also were able to show that Tsallis entropy can be used as a viable measure of dynamical complexity in the ionosphere, with portions showing lower values of Tsallis entropy indicating lower dynamical complexity, with a good relationship with Lyapunov exponents. They found a 
phase transition from a higher dimension during quiet days to a lower dimension during storm.

The low-latitude region where Nigeria is situated is known as the equatorial anomaly region; this region is known for the equatorial ionization anomaly, which is due to the fountain effect. Off the Equator, the E region electric field maps along the magnetic field up to the $\mathrm{F}$ region altitude in the low latitude; this eastward electric field (E) interacts with magnetic field $\mathrm{B}$ in the $\mathrm{F}$ region during the day. This results in the electrodynamic lifting of the $\mathrm{F}$ region plasma over the Equator, known as EXB drift. The uplifted plasma over the Equator moves along the magnetic line in response to gravity, diffusion and pressure gradients and, hence, the fountain effect. The fountain effect that is controlled by the EXB drift shows the dynamics of the diurnal variation equatorial anomaly (Abdu, 1997; Unnikrishnan, 2010). There is a reduction in the $\mathrm{F}$ region ionization density at the magnetic Equator and also much enhanced ionization density at the two anomaly crests within $\pm 15^{\circ}$ of the magnetic latitude north and south of the Equator (Rama Rao et al., 2006). The equatorial ionization anomaly and other natural processes which include various ionization processes and recombination, influx of solar wind, photoionization processes and so many other factors that occur due to variations in solar activities, have a great influence on the systems of the ionosphere, due to their effects on internal dynamics of the ionosphere. This portrays the ionosphere as a typical natural system with continuous interaction with its external environment, which led to the study of the influence of the Sun on the ionosphere (Ogunsua et al., 2014).

The ionosphere possesses a significant level of non-linear variations that requires more investigation, which can be studied and characterized using a non-linear approach like the chaoticity and dynamical complexity for the study of its dynamics. The need to study the daily variation in the dynamical complexity of the ionosphere arises from the established knowledge and understanding which shows that the ionosphere is a complex system with so many variations that can arise from various dynamical changes that can be due to various changes in different processes that contribute to the behaviour and nature of the ionosphere. Rabiu et al. (2007) affirmed that characterizing the ionosphere is of utmost importance due to the numerous complexities associated with the region. The scale of these numerous complexities interestingly changes at times from one day to another.

The concept of chaos as previously applied to ionospheric and magnetospheric studies on quiet and stormy conditions is limited. Most investigations have been based on quiet and storm conditions only for all studies carried out, and none of the previous works involved the use of quiet and disturbed day classification of geophysical conditions until recently by Ogunsua et al. (2014), where we considered the comparative use of Lyapunov exponents and Tsallis entropy as proxies for the internal dynamics of the ionosphere. This is the main
Table 1. Coordinates of the GPS stations.

\begin{tabular}{|c|c|c|c|}
\hline \multirow{2}{*}{$\begin{array}{l}\text { Station } \\
\text { name }\end{array}$} & \multicolumn{2}{|c|}{ Geographic coordinates } & \multirow{2}{*}{$\begin{array}{r}\text { Dip latitude } \\
\left({ }^{\circ} \mathrm{N}\right)\end{array}$} \\
\hline & Long. $\left({ }^{\circ} \mathrm{E}\right)$ & Lat. $\left({ }^{\circ} \mathrm{N}\right)$ & \\
\hline Birnin Kebbi & $\begin{array}{l}4^{\circ} 12^{\prime} \mathrm{E} \\
12^{\circ} 32^{\prime} \mathrm{N}\end{array}$ & & $0.62^{\circ} \mathrm{N}$ \\
\hline Torro & $\begin{array}{l}9^{\circ} 04^{\prime} \mathrm{E} \\
10^{\circ} 03^{\prime} \mathrm{N}\end{array}$ & & $-0.82^{\circ} \mathrm{N}$ \\
\hline Yola & $12^{\circ} 30^{\prime} \mathrm{E}$ & $9^{\circ} 12^{\prime} \mathrm{N}$ & $-1.39^{\circ} \mathrm{N}$ \\
\hline Lagos & $3^{\circ} 23^{\prime} \mathrm{E}$ & $6^{\circ} 27^{\prime} \mathrm{N}$ & $-3.07^{\circ} \mathrm{N}$ \\
\hline Enugu & $7^{\circ} 30^{\prime} \mathrm{E}$ & $6^{\circ} 26^{\prime} \mathrm{N}$ & $-3.21^{\circ} \mathrm{N}$ \\
\hline
\end{tabular}

reason for the consideration of day-to-day variation of these parameters in this work.

\section{Data and methodology}

The data used for this study are the Global Positioning System (GPS) total electron content (TEC) data obtained from five GPS satellite receiver stations. Table 1 shows the coordinates of the stations. These receivers take the measure of slant TEC within a $1 \mathrm{~m}^{2}$ columnar unit of the cross section along the ray path of the satellite and the receiver which is given by

STEC $=\int_{\text {receiver }}^{\text {Satellite }} \mathrm{Ndl}$.

The observation of the total number of free electrons along the ray path is derived from the frequencies $L_{1}$ $(1572.42 \mathrm{MHz})$ and $L_{2}(1227.60 \mathrm{MHz})$ of the Global Positioning System (GPS) that provide the relative ionosphere delay of electromagnetic waves travelling through the medium (Saito et al., 1998). The slant TEC is projected to vertical TEC using the thin shell model assuming a height of $350 \mathrm{~m}$ (Klobuchar, 1986):

$\mathrm{VTEC}=\mathrm{STEC} \cdot \cos \left[\arcsin \left(R_{\mathrm{e}} \cos \Theta / R_{\mathrm{e}}+h_{\max }\right)\right]$,

where $R_{\mathrm{e}}=6378 \mathrm{~km}$ (radius of the Earth), $h_{\max }=350 \mathrm{~km}$ (the vertical height assumed from the satellite) and $\Theta$ is the elevation angle at the ground station.

In this study, five GPS TEC measuring stations lying within the low-latitude region were considered, as shown in Table 1. The TEC data obtained for January to December 2011 were considered for this study and the data are given at $1 \mathrm{~min}$ sampling time. The TEC data were subjected to various analyses which will be discussed in the next section. The day-to-day variations of the chaotic behaviour and dynamical complexity were studied for the entire year. The surrogate data tests for non-linearity were also conducted for both the dynamical and geometrical aspects. 


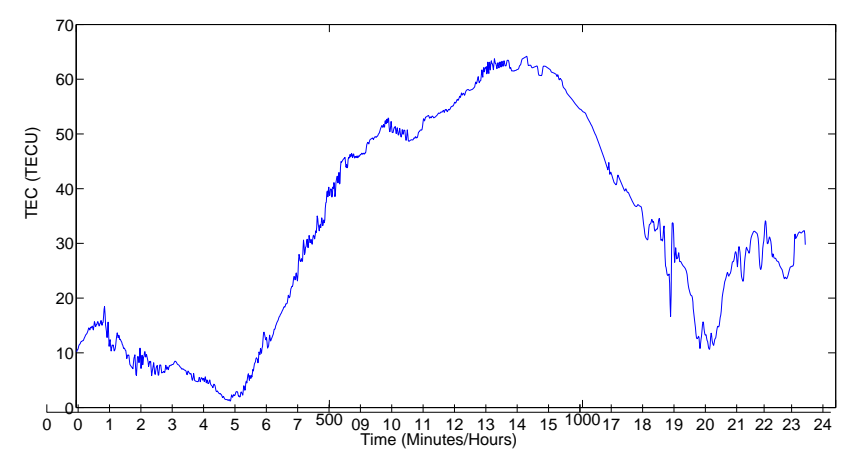

Figure 1. A typical time series plot for TEC measured at Lagos for 20 November 2011.

\section{Methods of data analysis and results}

\subsection{Time series analysis}

A time series can be seen as a numerical account that describes the state of a system from which it was measured. A given time series, $S_{n}$, can be defined as a sequence of scalar measurement of a particular quantity taken as a series at different portions in time for a given time interval $(\Delta t)$. The time series describes the physical appearance of an entire system, as seen in Fig. 1. However, it may not always describe the internal dynamics of that system. A system like the ionosphere possesses a dominant dynamics that can be seen as diurnal, so the data should be treated so as to be able to see its internal dynamics. The measured TEC time series were plotted to see the dynamics of the system. A typical plot of TEC usually has a dominant dynamics (see Fig. 1) which may be seen as the diurnal behaviour; however, it can also be seen that there is a presence of fluctuations (which appear to be non-linear) in the system as a result of the internal dynamics of the ionosphere and space plasma system, due to different activities in the ionosphere. Therefore, there is a need to minimize the influence of the diurnal variations since we are more interested in the non-linear internal dynamics of the system in this study; to do so, the TEC time series was detrended by carrying out the following analysis.

Since for the given daily data of 1 min sampling time there are 1440 data points per day, there exists a time series $t_{i}$ where $i=1,2,3 \ldots 1440$ represents the observed time series, and there also exists a set of $u_{i}$ where $i=1,2,3 \ldots 1440$, such that the diurnal variation reduced time is given by

$T_{i}=t_{i}-u_{j}$,

where $i=1,2,3, \ldots, j=\bmod (i, 1440)$ if $\bmod (j, 1440) \neq 0$ and $j=1440$ if $d(j, 1440)=0$. This method will give the detrended time series represented by $T_{i}$ obtained from the original TEC data as shown in Fig. 2. This method is similar to that used by Unnikrishnan et al. (2006) and Unnikrishnan (2010); the further explanations of the dynamical results

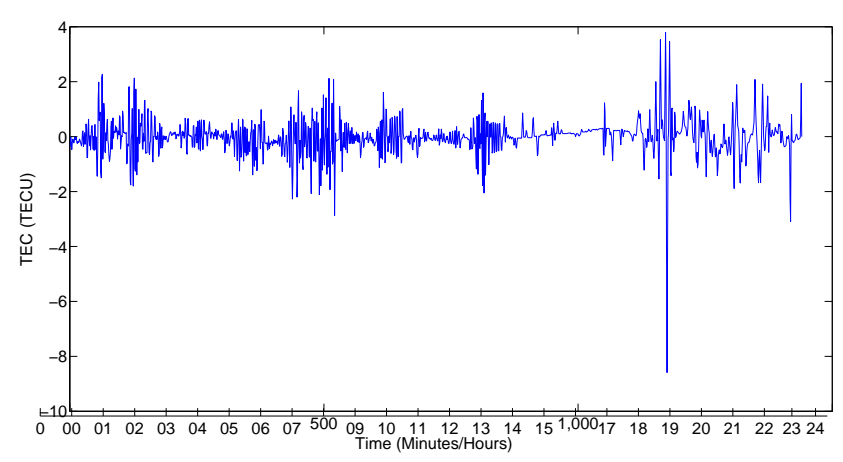

Figure 2. The detrended time series plot for TEC measured at Lagos.

can be found in Kumar et al. (2007). The detrended time series were subjected to further analyses for the phase space reconstruction and also to obtain the values of Lyapunov exponents, correlation dimension, Tsallis entropy and the implementation of a surrogate data test.

\subsubsection{Phase space reconstruction and non-linear time series analysis}

The study of chaoticity and dynamical complexity in a dynamical system requires a non-linear approach, due to the fact that systems described by these phenomena can be referred to as non-linear complex systems. The magnetosphere and the ionosphere are good examples of such systems. To be able to study such phenomena, some non-linear time series analysis can be carried out on the time series data describing such a system. The detrended time series of TEC measurement is subjected to some non-linear time series data analysis to obtain the mutual information and false nearest neighbours, embedding dimension and delay coordinates for the phase space reconstruction, and the evaluation of other chaotic quantifiers, namely, Lyapunov exponents, correlation dimension, recurrence analysis, and entropy.

The phase space reconstruction helps to reveal the multidirectional aspect of the system. The phase space reconstruction is based on the embedding theorem, such that the phase space is reconstructed to show the multidimensional nature as follows:

$\boldsymbol{Y}_{n}=\left(s_{n}-(m-1) \tau s_{n}-(m-2) \tau \ldots s_{n}-\tau s_{n}\right)$,

where $\boldsymbol{Y}_{n}$ are vectors in phase space. The proper choice of embedding dimension $(m)$ and delay time $(\tau)$ is essential for phase space reconstruction (Fraser and Swinney, 1986; Kennel et al., 1992).

If the plot showing the time-delayed mutual information shows a marked minimum, that value can be considered to be an optimal time delay; Fig. 3 shows the mutual information plotted against the time delay. Likewise, the minimal embedding dimension, which corresponds to the minimum 


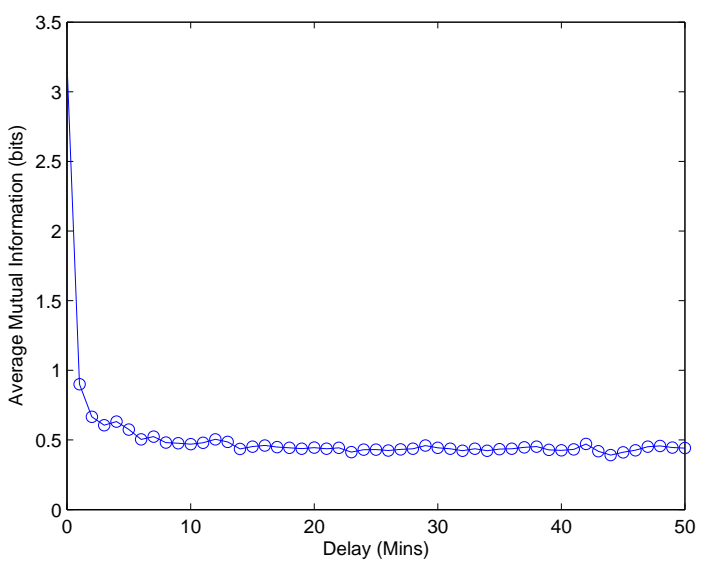

Figure 3. Average mutual information against time delay for TEC measured at Yola.

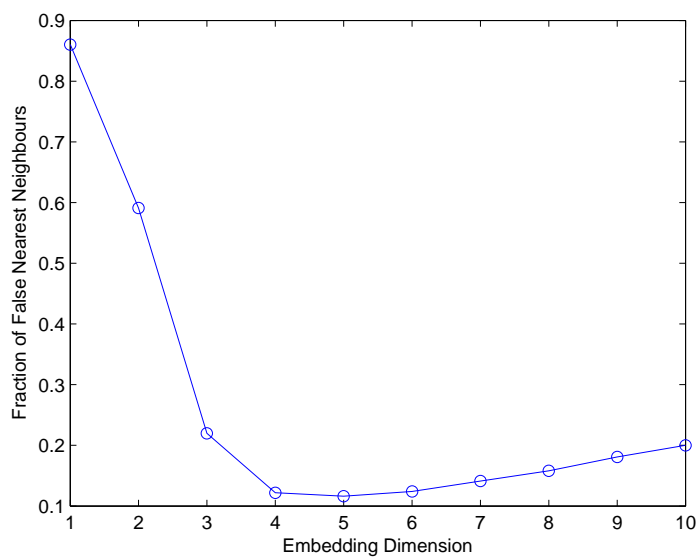

Figure 4. Fraction of false nearest neighbours against embedding dimension for TEC measured at Yola.

number of false nearest neighbours, can be treated as the optimum value of the embedding dimension in Unnikrishnan et al. (2006) and Unnikrishnan (2010). A plot of the fraction of false nearest neighbours against the embedding dimension can be seen in Fig. 4. It was observed that for all the daily detrended TEC time series, the choice of $\tau \geq 30$ and $m \geq 4$ values of delay and embedding dimension above these values is suitable for analysis of data for all stations. The choice of $\tau=30$ and $m=5$ was mostly used to analyse the dynamical aspects for all the stations. The reconstructed phase space trajectory is shown in Fig. 5.

\subsubsection{Lyapunov exponents}

The Lyapunov exponent has been a very important quantifier for the determination of chaos in a dynamical system. This quantifier is also used for the determination of chaos in time series, representing natural systems like the ionosphere and magnetosphere (Unnikrishnan, 2008, 2010). A positive Lyapunov exponent indicates divergence of trajec-

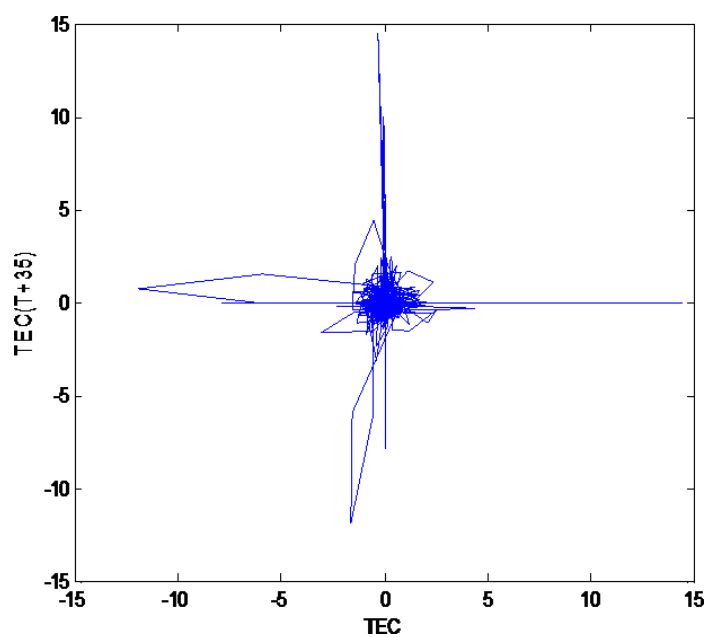

Figure 5. The delay representation of the phase space reconstruction of the detrended TEC.

tory in one dimension, or alternatively an expansion of volume, which can also be said to indicate repulsion or attraction from a fixed point. A positive Lyapunov exponent indicates that there is evidence of chaos in a dissipative deterministic system, where the positive Lyapunov exponent indicates divergence of trajectory in one direction or expansion of value, and a negative value shows convergence at trajectory or contraction of volume along another direction.

The largest Lyapunov exponent $\left(\lambda_{1}\right)$ can be used to determine the rate of divergence as indicated by Wolf et al. (1985), where

$\lambda_{1}=\frac{1}{t} \ln \frac{\Delta x(t)}{x(0)}=\frac{1}{t} \sum_{i=1}^{t} \ln \left(\frac{\Delta x\left(t_{i}\right)}{\Delta x\left(t_{i-1}\right)}\right)$.

The Lyapunov exponent was computed for the TEC values measured from different stations. The evolution in state space was scanned with $\tau=30$ and $m=5$, as shown in Fig. 6 . The day-to-day variations of the Lyapunov exponent were computed for the entire year so as to study the annual trend of variation. This was implemented using the method introduced by Rosenstein (1993) and Hegger et al. (1994); both algorithms use very similar methods. Lyapunov exponents were also computed for varying time delays at a constant embedding dimension and also for varying embedding dimension, to check for the stability with changes in trajectory. These can be seen in Fig. 6b and c. The day-to-day values of Lyapunov exponents plotted for the Enugu station and for the Toro station are shown in Fig. 7a-b. The plots of the dayto-day values show the transient variation of the ionosphere and a wavelike yearly pattern.

\subsubsection{Correlation dimension}

Another relevant method to study the underlying dynamics or internal dynamics of a system is to evaluate the dimension 

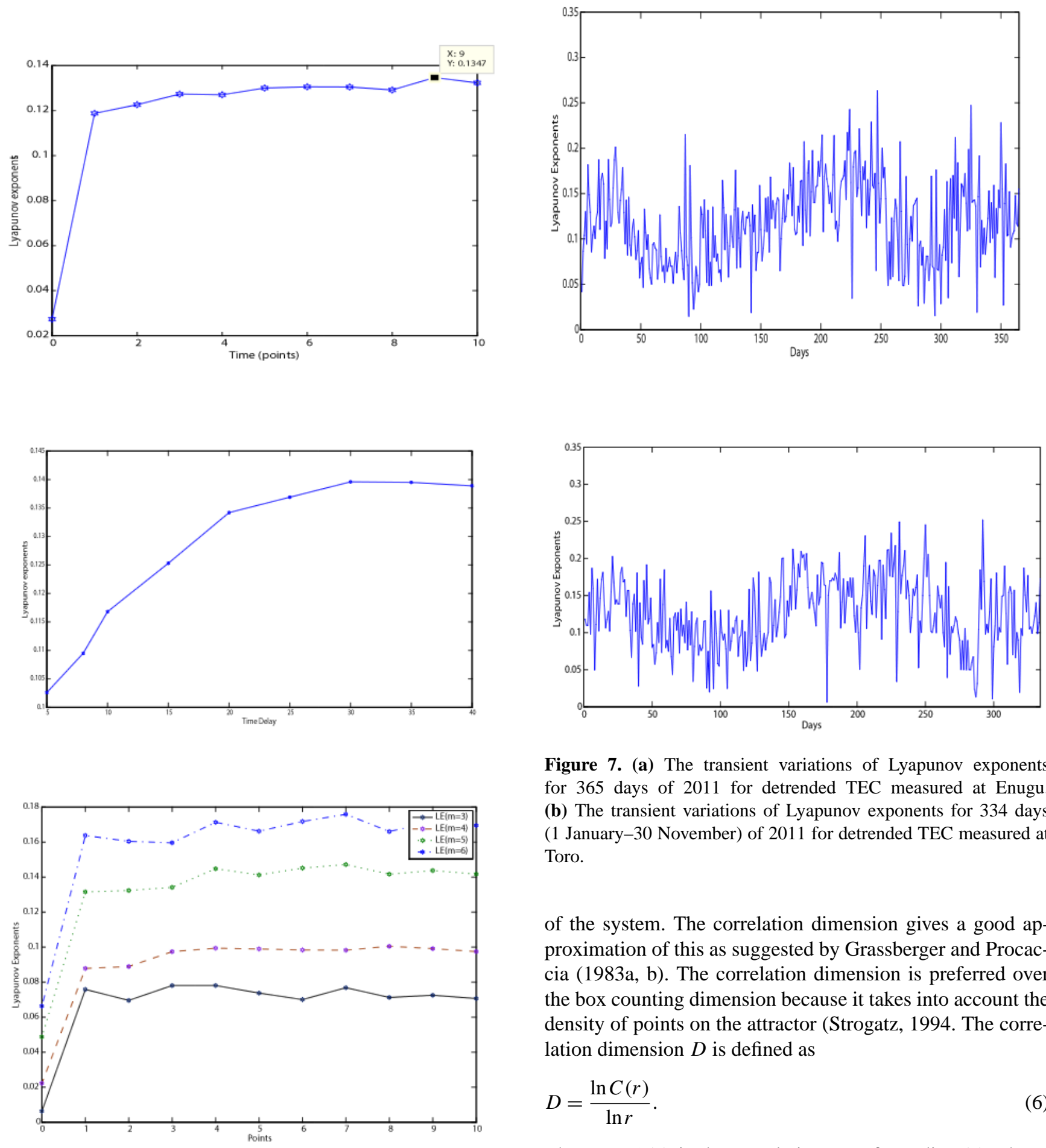

Figure 7. (a) The transient variations of Lyapunov exponents for 365 days of 2011 for detrended TEC measured at Enugu. (b) The transient variations of Lyapunov exponents for 334 days (1 January-30 November) of 2011 for detrended TEC measured at Toro.

of the system. The correlation dimension gives a good approximation of this as suggested by Grassberger and Procaccia $(1983 \mathrm{a}, \mathrm{b})$. The correlation dimension is preferred over the box counting dimension because it takes into account the density of points on the attractor (Strogatz, 1994. The correlation dimension $D$ is defined as

$D=\frac{\ln C(r)}{\ln r}$.

Figure 6. (a) Lyapunov exponent computed and its evolution computed as the state space trajectory scanned with $\tau=30, m=5$ for detrended time series measured at Yola with the largest Lyapunov exponent equal to 0.1347. (b) Lyapunov exponent computed for a different time delay with a constant embedding dimension. (c) Lyapunov exponents (LE) computed for different embedding dimensions ( $m=3,4,5$ and 6$)$ at a constant time delay.

The term $C(r)$ is the correlation sum for radius $(r)$ where, for a small radius $(r)$, the correlation sum can be seen as $C(r) \sim r^{d}$ for $r \rightarrow 0$. The correlation sum is dependent on the embedding dimension $(m)$ of the reconstructed phase space and is also dependent on the length of the time series $N$ as follows:

$C(r)=\frac{2}{N(N-1)} \sum_{i=1}^{N} \sum_{j=i+1}^{N} \Theta\left(r-\left|y_{i}-y_{j}\right|\right)$, 
where $\Theta$ is the Heaviside step function, with $\Theta(H)=0$ if $H \leq 0$ and $\Theta(H)=1$ for $H>0$.

The correlation dimension was computed using the Theiler algorithm approach, with the Theiler window $(w)$ at 180 . The Theiler window was chosen to be approximately equal to the product of $m$ and $\tau$. A similar approach to the computation of the correlation dimension was used by Unnikrishnan and Ravindran (2010) to determine the correlation dimension of detrended TEC data for some stations in India, which lies within the equatorial region, like Nigeria. Ogunsua et al. (2014) also used similar methods for some detrended TEC from Nigerian stations.

The correlation dimensions for data taken for the quietest day of October 2011 and the most disturbed day of October 2011 from the Birnin Kebbi GPS TEC measuring station were represented by Fig. 8a and b respectively. The correlation dimension saturates at $m \geq 4$ for the quietest day of the month and at $m \geq 5$ for the most disturbed day. In this illustration, the most disturbed day of this month falls within the storm period of October 2011. The classification of days into quiet and disturbed days in the month of October 2011 enables us to compare the quiet and storm periods together while comparing the quiet days with some relatively disturbed days.

\subsubsection{Computation of Tsallis entropy and principles of non-extensive Tsallis entropy}

Entropy measures are very important statistical techniques that can be used to describe the dynamical nature of a system. The Tsallis entropy can be used to describe the dynamical complexity of a system and also to understand the nonlinear dynamics like chaos which may exist in a natural system. The use of entropy measures as a method to describe the state of a physical system has been employed in information theory for decades. The computation of entropy allows us to describe the state of disorderliness in a system; one can generalize this same concept to characterize the amount of information stored in more general probability distributions (Kantz and Shrieber, 2003; Balasis et al., 2009). The concept of information theory is basically concerned with these principles. The information theory gives us an important approach to time series analysis. If our time series, which is a stream of numbers, is given as a source of information such that these numbers are distributed according to some probability distribution, then transitions between numbers occur with well-defined probabilities. One can deduce the same average behaviour of the system at a different point and for the future. The term entropy is used in both physics and information theory to describe the amount of uncertainty or information inherent in an object or system (Kantz and Schrieber, 2003). The state of an open system is usually associated with a degree of uncertainty that can be quantified by the Boltzmann-Gibbs entropy, a very useful uncertainty measure in statistical mechanics. However, Boltzmann-Gibbs
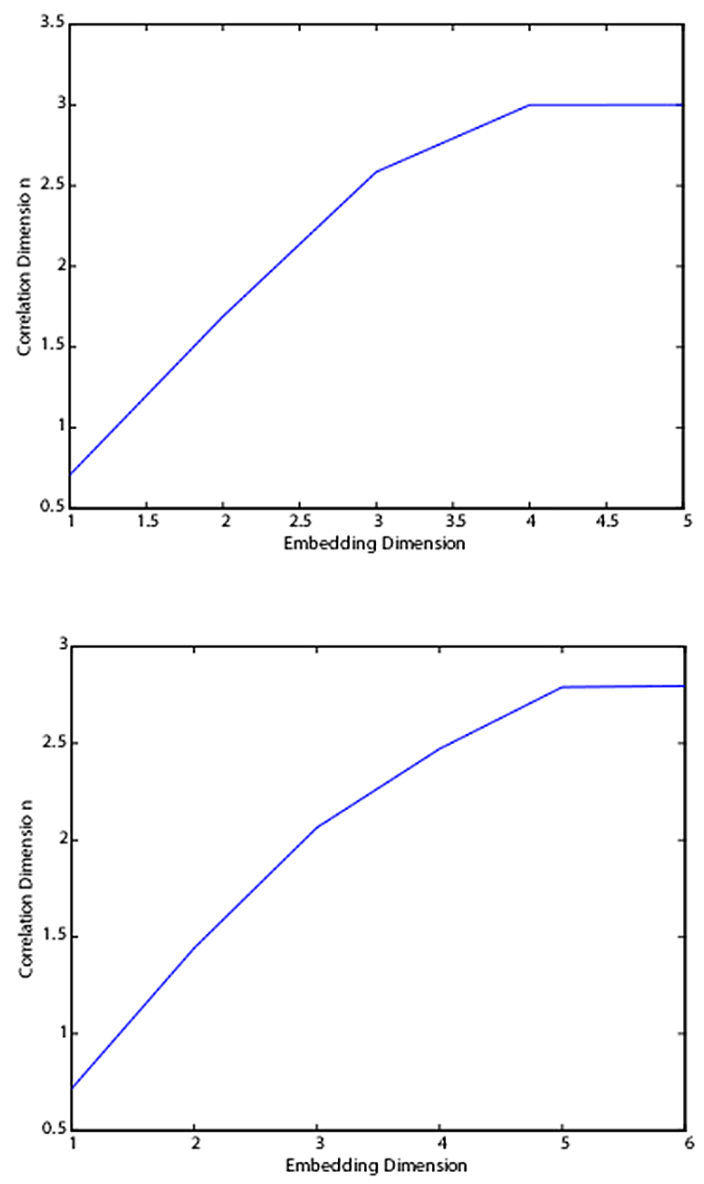

Figure 8. (a) The correlation dimension of the detrended TEC for the quietest day of October at Birnin Kebbi, which saturates at $m \geq 4$ and $\tau=39$. (b) The correlation dimension of the detrended TEC for the most disturbed day of October at Birnin Kebbi, which saturates at $m \geq 5$ and $\tau=34$.

entropy cannot describe non-equilibrium physical systems with large variability and a multifractal structure such as the solar wind (Burgala et al., 2007; Balasis et al., 2008). One of the crucial properties of the Boltzmann-Gibbs entropy in the context of classical thermodynamics is extensivity, namely, proportionality with the number of elements of the system. The Boltzmann-Gibbs entropy satisfies this prescription if the subsystems are statistically (quasi-)independent, or typically if the correlations within the system are essentially local. In such cases the system is called extensive. In general, however, the situation is not of this type and correlations may be far from negligible at all scales. In such cases, the Boltzmann-Gibbs entropy is non-extensive (Balasis et al., 2008, 2009). These generalizations above were proposed by Tsallis (1988), who was inspired by the probabilistic description of multifractal geometries. Tsallis $(1988,1998)$ introduced an entropy measure by presenting an entropic expression characterized by an index $q$ which leads to nonextensive statistics, 
$S_{q}=k \frac{1}{q-1}\left(1-\sum_{i=1}^{W} p_{i}^{q}\right)$,

where $p_{i}$ are the probabilities associated with the microscopic configurations, $W$ is their total number, $q$ is a real number, and $k$ is Boltzmann's constant. The value $q$ is a measure of the non-extensitvity of the system: $q \rightarrow 1$ corresponds to the standard extensive Boltzmann-Gibbs statistics. This is the basis of the so-called non-extensive statistical mechanics, which generalizes the Boltzmann-Gibbs theory. The entropic index $q$ characterizes the degree of nonadditivity reflected in the following pseudoadditivity rule:

$$
\begin{aligned}
\frac{S_{q}(A+B)}{k} & =\left[\frac{S_{q}(A)}{k}\right]+\left[\frac{S_{q}(B)}{k}\right] \\
& +(1-q)\left[S_{q}(A) / k\right]\left[\frac{S_{q}(B)}{k}\right] .
\end{aligned}
$$

The cases $q>1$ and $q<1$ correspond to subadditivity (or subextensivity) and superadditivity (or superextensivity) respectively, and $q=1$ represents additivity (or extensivity). For subsystems that have special theory probability correlations, extensivity is not valid for Boltzmann-Gibbs entropy in such cases, but may occur for $S_{q}$ with a particular value of the index $q$. Such systems are sometimes referred to as non-extensive (Boon and Tsallis, 2005; Balasis et al., 2008, 2009). The parameter $q$ itself is not a measure of the complexity of the system, but measures the degree of non-extensivity of the system. It is the time variations of the Tsallis entropy for a given $q\left(S_{q}\right)$ that quantify the dynamic changes of the complexity of the system. Lower $S_{q}$ values characterize the portions of the signal with lower complexity. In this presentation we estimate $S_{q}$ on the basis of the concept of symbolic dynamics and by using the technique of lumping (Balasis et al., 2008, 2009).

A comparison of Tsallis entropy with Lyapunov exponents computed for the same set of data has been carried out in this work, to see the efficacy of the combined usage of both parameters. This is based on the established facts that variations in the values of Tsallis entropy can be linked with that of Lyapunov exponents' chaotic behaviour in systems as seen in Baranger et al. (2012), Anastasiadis et al. (2005), and Kalogeropoulos et al. (2012, 2013). Coraddu et al. (2005) showed the Tsallis entropy generalization for Lyapunov exponents. Further details can be found in Ogunsua et al. (2014); they were able to investigate the similarities in their response to the complex dynamics of the ionosphere, and this informs the further use of the two quantities as indices to study the day-to-day variation of ionospheric behaviour in this work.

The values of these entropy measures were also computed in order to study the dynamical complexity of the system under observation (the ionosphere). The day-to-day values of Tsallis entropy were computed for the entire year for different stations. The day-to-day values of Tsallis entropy plotted
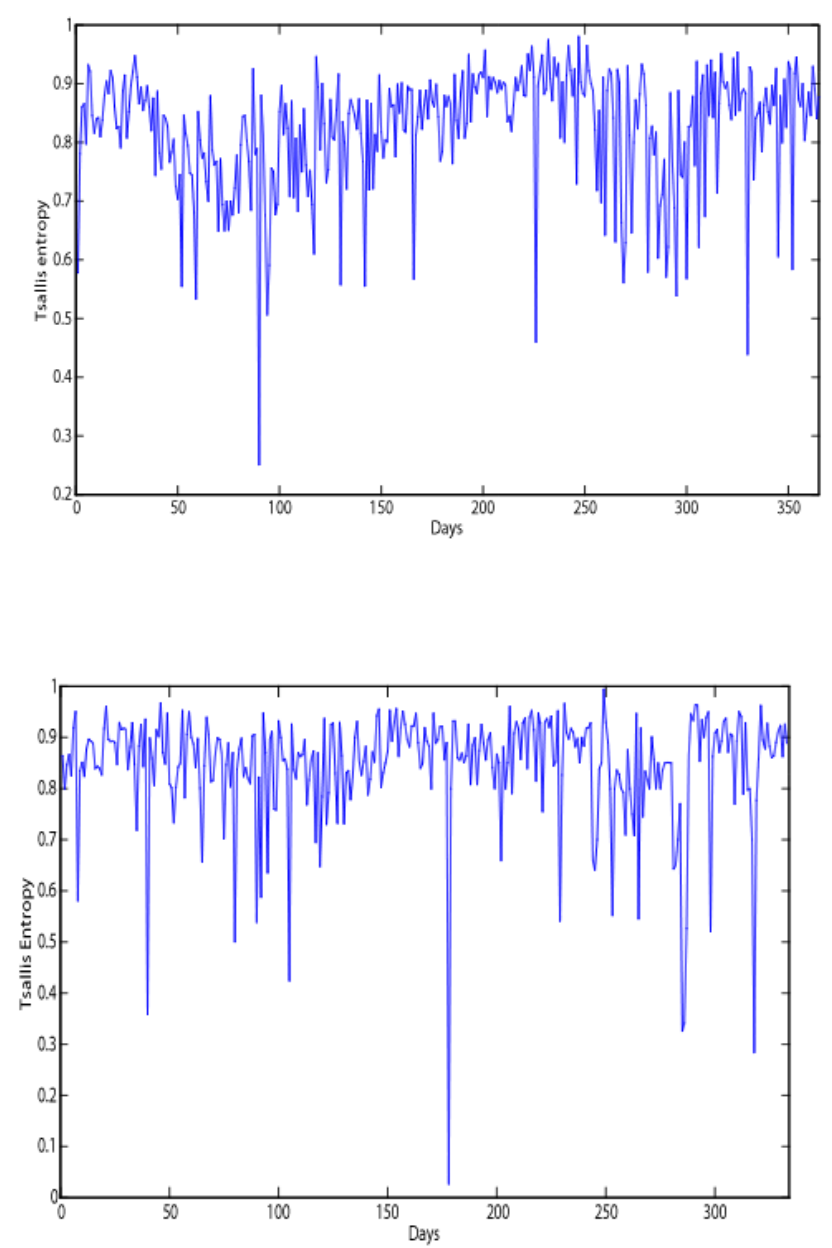

Figure 9. (a) The transient variations of Tsallis entropy for 365 days (1 January-30 November) of 2011 for detrended TEC measured at Enugu. (b) The transient variations of Tsallis entropy for 334 days (1 January-30 November) of 2011 for detrended TEC measured at Toro.

for the Enugu station and for the Toro station are shown in Fig. 9a and $\mathrm{b}$. The plots of the day-to-day values show the transient variation of the ionosphere and a wavelike yearly pattern.

\subsection{Non-linearity test using surrogate data}

The test for non-linearity using the method of surrogate data according to Kantz and Schreber (2003) has proven to be a good test for non-linearity in time series describing a system. It has been accepted that the method of a surrogate data test could be a successful tool for the identification of a nonlinear deterministic structure in experimental data (Pavlos et al., 1999). This method involves creating a test of significance of difference between a linearly developed surrogate and original non-linear time series to be tested. The test is done by carrying out the computation of the same quantity on both surrogates and the original time series and then check- 


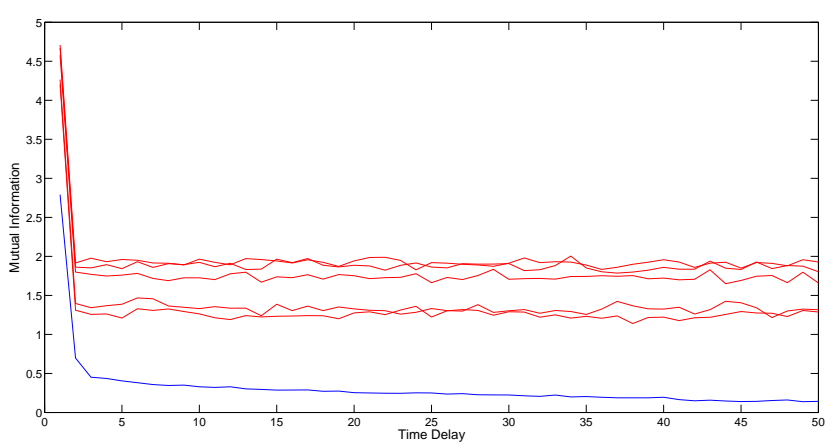

Figure 10. Mutual information plotted against time delay for the original detrended data (blue curve) with the mutual information for the surrogate data (red curve) for TEC data measured at Lagos for the quietest day of March 2011.

ing for the significance of difference between the results obtained from the surrogates with the original data. Theiler et al. (1992) suggested the creation of surrogate data by using Monte Carlo techniques for accurate results. According to this method, typical characteristics of data under study are compared with those of stochastic signals (surrogates), which have the same auto-correlation function and the power spectrum of the original time series. It can be safely concluded from the test of significance carried out on the surrogate and the original data that a stationary linear Gaussian stochastic model cannot describe the process under study provided that the behaviour of the original data and the surrogate data are significantly different.

In this work, 10 surrogate data were generated from the original data set. The geometrical and dynamical characteristics of the original data were then compared to those of the surrogates using the statistical method of significance of difference, which can be defined as

$S=\frac{\alpha_{\text {Surr }}-\alpha_{\text {Original }}}{\sigma}$,

where $\alpha_{\text {Surr }}$ is the mean value of the computed quantity for the surrogate data and $\alpha_{\text {Original }}$ is the same quantity computed for the original TEC data; $\sigma$ is the standard deviation of the same quantity computed for the surrogate data. The significance of difference considered for the null hypothesis to be rejected here is greater than 2 , which enables us to be able to reject the null hypothesis that the original TEC data describing the ionospheric system can be modelled using a Gaussian linear stochastic model with confidence greater than $95 \%$.

The surrogate data test for all stations used in this study shows the Lyapunov exponents of the surrogate data for the selected days in October shown in the table below; the results show that the surrogate data test for Lyapunov exponents shows a significance of difference greater than 2 for all the selected days for all the stations. Similar results were obtained for mutual information, fraction of false nearest neighbours, and correlation dimension. This result gives us the

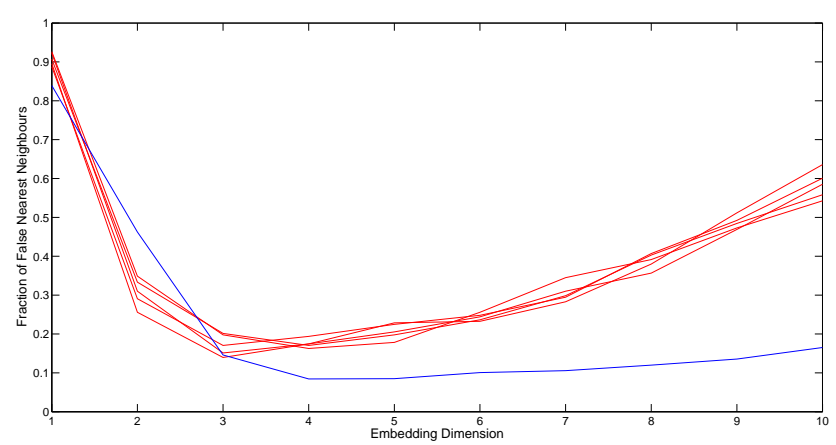

Figure 11. Fraction of false nearest neighbours plotted against the time embedding dimension for the original detrended data (blue curve) with the mutual information for the surrogate data (red curve) for TEC data measured at Lagos for the quietest day of 20 March.

Table 2. (a) Results of surrogate data tests for Lyapunov exponents for TEC data for the quietest days of October 2011 at Birnin Kebbi station. (b) Results of surrogate data tests for Lyapunov exponents for TEC data for the most disturbed days of October 2011 at Birnin Kebbi station.

\begin{tabular}{cc}
\hline Original data & Surrogate data \\
\hline & (a) \\
\hline 0.1165 & $0.3921 \pm 0.0420$ \\
0.0931 & $0.2029 \pm 0.0756$ \\
0.1041 & $0.3860 \pm 0.0741$ \\
0.0498 & $0.2891 \pm 0.0598$ \\
0.1420 & $0.3621 \pm 0.0504$ \\
\hline & (b) \\
\hline 0.0579 & $0.3039 \pm 0.0541$ \\
0.0502 & $0.3156 \pm 0.0428$ \\
0.0786 & $0.2527 \pm 0.0296$ \\
0.1795 & $0.3662 \pm 0.0468$ \\
0.1038 & $0.3100 \pm 0.0416$ \\
\hline
\end{tabular}

confidence to reject the null hypothesis that the data used cannot be modelled using a linear Gaussian stochastic model, which shows that the system is a non-linear system with some level of determinism. Figure 10 shows the plots comparing the mutual information plotted against the time delay for the original detrended data (blue curve) with the mutual information for the surrogate data for TEC data measured at Lagos for the quietest day of March 2011, while Fig. 11 compares the fraction of false nearest neighbours for the same set of data. Table 2 a shows the values of Lyapunov exponents for both original detrended and surrogate data for TEC measured in Lagos during the quietest days and Table $2 \mathrm{~b}$ shows the values of Lyapunov exponents for both original detrended and surrogate data for TEC measured in Lagos during the most disturbed days of October 2011. The result obtained from the surrogate data test shows that we cannot represent the original detrended data with a linear Gaussian stochastic model. 


\subsection{Trend filtering using the moving average approach for the daily values}

The trend of a fluctuating time series can be made clearer to reveal the general pattern of that time series, and to make the fluctuating pattern of the daily variation of the chaoticity and dynamical complexity measures clearer in the work, the moving average method has been employed. The method of moving average filtering has found its applications in geophysics (e.g. Bloomfeld, 1992; Bloomfield and Nychka, 1992; Baillie and Chung, 2002) and in other areas like financial time series analysis, microeconomics, biological sciences and medical sciences. The various fields mentioned require different trend filtering methods, depending on the structure of the time series to be analysed. Different filtering processes that can be used to reveal the trend include the moving average filters, exponential filters, band-pass filtering, median filtering, etc.

Suppose we have a time series $z[t]$ such that $t=1,2,3 \ldots n$, where $n$ could assume any value, if $z[t]$ consists of a consistently varying trend component that appears over a longer period of time $t$ given as $u[t]$ and a more rapidly varying component $v[t]$. The goal of trend filtering in any research is to estimate either of the two components (Kim et al., 2009). The purpose of trend filtering in this work is to further reveal the general slow varying trend that appears to be obvious in the daily variation of the values of the chaoticity and dynamical complexity of the ionosphere, which might appear to be obviously varying with the yearly solar activity (a quantity with a slow varying trend). To make $u[t]$ (which represents the general slow varying trend) smoother and in the process reduce $v[t]$, we apply the moving average filter.

If we assume $z[t]$ to be our time series representing the daily variation of the values of the chaoticity and dynamical complexity of the ionosphere, then our smoothing with weighting vector/filter $w_{j}$ will create the new sequence $u_{j}$ as

$u[t]=z[t] \cdot w[n]=\frac{1}{2 k+1} \sum_{i=-k}^{k} x[n-1]$.

In this work, the Savitzy-Golay method of smoothing proposed by Savitzky and Goley (1967), which is a generalized form of the moving average, was applied to the trend smoothing of the daily variation of the chaoticity and dynamical complexity of the ionosphere. In this case it performs a least square fit to a small set of $L(=2 k+1)$ consecutive data to a polynomial and then takes the midpoint of the polynomial curve as output. The smoothed time series in this work will now be given as $u[t]=z[t] \cdot \omega[n]=\frac{\sum_{i=-k}^{k} A_{i} \cdot x[n-1]}{\sum_{i=-k}^{k} A_{i}}$,

where $\omega[n]=\frac{A_{n}}{\sum_{i=-k}^{k} A_{i}}-k \leq n \leq k$ such that $A_{i}$ controls the order of the polynomial. A similar method was described in Reddy et al. (2010).

The smoothed daily variation and the original data and the plot of the smoothed variation only, for the Lyapunov exponents of the detrended TEC measured at the Enugu and Toro stations, are shown in Fig. 12a and b. The smoothed day-today variation for Tsallis entropy for the detrended TEC measured at the Enugu and Toro stations respectively is shown in Fig. 13a and $b$.

\section{Discussion}

The results presented in the work reveal the dynamical characteristics of the ionosphere. These characteristics are discussed in this section, considering the time series treatment and phase space reconstruction; the study of chaos uses chaotic quantifiers and the use and comparison of dynamical complexity measures in terms of their response to the variations in ionospheric dynamics. Also discussed is the implication of the non-linearity test using the surrogate data and the comparison of the two quantifiers and their viability as indices for the continuous study and characterization of the ionosphere.

The time series analysis shows the appearance of some degree of non-linearity in the internal dynamics of the ionosphere. The time series plot in Fig. 1 shows the rise in TEC to peak at the sunlit hours of the day; however, it can be seen that the rise to the peak exhibited by the ionosphere, which is the dominant dynamics during the day, makes it impossible to clearly see the internal dynamics of the system from the TEC time series plot. It can be seen that the TEC time series curve is not a smooth curve with tiny variations, which probably describes a part of the internal dynamics. These visible tiny variations around the edges of the time series plot can be regarded as the rate of change of TEC, which is a phenomenon that can describe the influence of scintillations in the ionosphere; these variations are however more obvious during the nighttime between the 1100th and 1440th minutes of the day (that is, between about 18:00 and 24:00 of the day). It should be noted here that scintillation has been described as a nighttime phenomenon associated with spread $\mathrm{F}$, and it occurs around the pre-midnight and post-midnight periods (Vyas and Chandra, 1994; Vyas and Dayanandan, 2011; Mukherjee et al., 2012; Bhattacharyya and Pandit, 2014). The detrended data show the internal dynamics of the system more clearly, with a pattern similar to the values around 

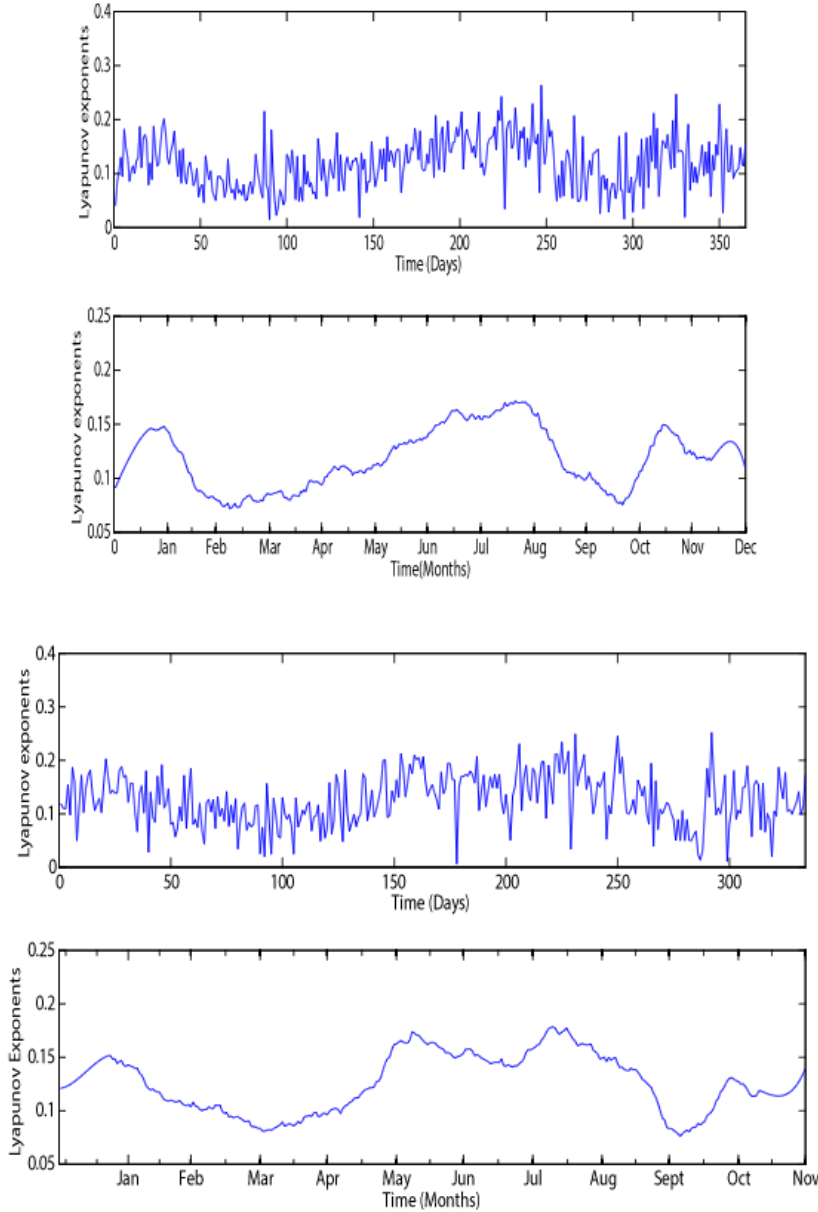

Figure 12. (a) Daily variation of Lyapunov exponents for TEC measured at the Enugu station for the year 2011 showing the original data (upper panel) and the smoothed plot of daily variation of Lyapunov exponents for TEC measured at the Enugu station for the year 2011 (lower panel). (b) Daily variation of Lyapunov exponents for TEC measured at the Toro station for the year 2011 showing the original data (upper panel) and the smoothed plot of daily variation of Lyapunov exponents for TEC measured at the Toro station for the year 2011 (lower panel).

the night period mentioned earlier. The post-sunset values (especially at nighttime) in Fig. 1 show a similar pattern to the detrended TEC plot in Fig. 2. It has been established that TEC does not decrease totally throughout the night as expected normally through the simple theory that TEC builds up during the day, but it shows some anomalous enhancements and variations, and this can occur over a wide range of geophysical conditions (Balan and Rao, 1987; Balan et al., 1991; Unnikrishnan and Ravindran, 2010). The delay representation of the phase space reconstruction shows a trajectory that is clustered around its origin, for all the stations, which can be seen as an indication of the possible presence of chaos. The degree of closeness of these trajectories however varies for different days from one station to another, resulting from
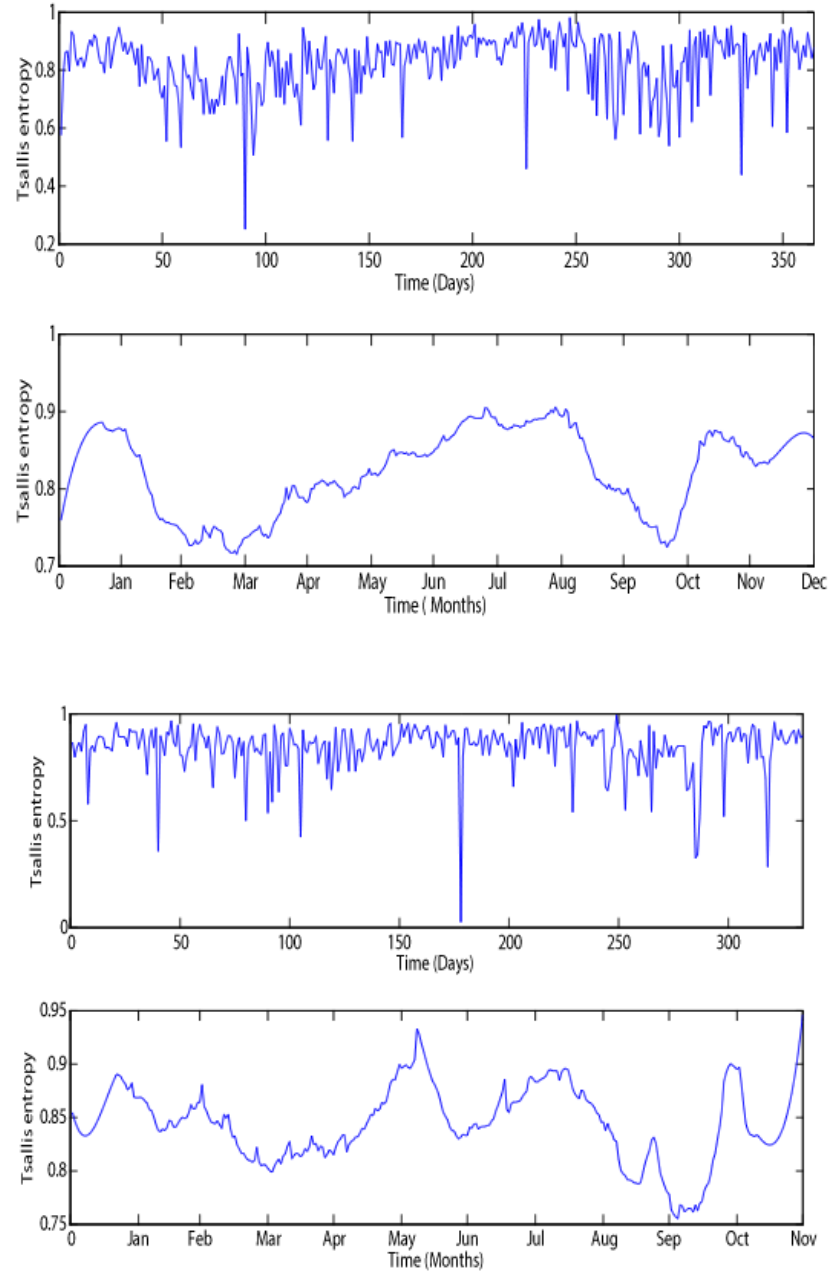

Figure 13. (a) Daily variation of Tsallis entropy for TEC measured at the Enugu station for the year 2011 showing the original data (upper panel) and the smoothed plot of daily variation of Lyapunov exponents for TEC measured at the Enugu station for the year 2011 (lower panel). (b) Daily variation of Tsallis entropy for TEC measured at the Toro station for the year 2011 showing the original data (upper panel) and the smoothed plot of daily variation of Lyapunov exponents for TEC measured at the Enugu station for the year 2011 (lower panel).

varying degrees of variations in stochasticity and determinism. The varying degrees of variations in stochasticity and determinism can be attributed to the daily variations and local time variations of photoionization, recombination, influx of solar wind and other factors that may influence the daily variations of TEC (Unnikrishnan, 2010).

The positive values of Lyapunov exponents indicate the presence of chaos (Wolf et al., 1985; Rosenstein et al., 1993; Hegger et al., 1999; Kantz and Schreiber, 2003). The presence of chaos was revealed by the positive Lyapunov exponents computed from all stations, and this is a result of the fact that the ionosphere is a system controlled by many parameters influencing its internal dynamics. Because of its ex- 
treme sensitivity to solar activity, the ionosphere is a very sensitive monitor of solar events. The ionospheric structure and peak densities in the ionosphere vary greatly with time (sunspot cycle, seasonally and diurnally), with geographical location (polar, auroral zones, mid-latitudes, and equatorial regions), and with certain solar-related ionospheric disturbances. During and following a geomagnetic storm, the ionospheric changes around the globe, as observed from the ground site, can appear chaotic (Fuller-Rowell et al., 1994; Cosolini and Chang, 2001; Unnikrishnan and Ravindran, 2010). The recorded presence of chaos as indicated by the positive values of Lyapunov exponents was found in all the computations, for all the TEC values obtained for the selected days from all the measuring stations used in this work. This can be expected, as it agrees with results from previous works that show that there is a reasonable presence of chaos in the ionosphere, even in the midst of the influence of stochastic drivers like solar wind (Bhattacharyya, 1990; Wernik and Yeh, 1994; Kumar et al., 2007; Unnikrishnan et al., 2006a, b; Unnikrishnan, 2010). However, the values of Lyapunov exponents vary from day to day due to variations in ionospheric processes for different days at the same latitude, as seen in Fig. 7a and b, with Fig. 12a and b showing the day-to-day variation (upper panel) and the smoothed curve of the day-to-day variation (lower panel) for the entire year. There are also latitudinal variations due to spatial variations in the various ionospheric processes taking place simultaneously. The ionosphere is said to have a complex structure due to these varying ionospheric processes.

The higher values of Lyapunov exponents during months of low solar activity (the solstices) is evidence that the rate of exponential growth in infinitesimal perturbations in the ionosphere leading to chaotic dynamics might be of a higher degree during most of the days of those months compared to days of the months with high solar activities showing lower values of Lyapunov exponents (Unnikrishnan, 2010; Unnikrishnan and Ravindran, 2010).

The results of the correlation dimension values computed are within the range of 2.7 to 3.2, with the lower values occurring mostly during the storm periods. The lower dimension during the storm periods compared to the quiet days may be due to the effect of a stochastic driver that occurs during geomagnetic storms on the internal dynamics of the ionosphere, like strong solar wind and solar flares; this could have been as a result of the fact that the internal dynamics must have been suppressed by the external influence. The restructuring of the internal dynamics of the ionosphere might be responsible for low-dimension chaos during storms and also the lower values of other measures like the Lyapunov exponents. The relatively disturbed day however might have a higher dimension so long as it is not a storm period, and sometimes a relatively disturbed day of the month might be a day with storm, and in this case there is usually a lower value of chaoticity and sometimes lower values of correlation dimension as well. The lower value of chaoticity and dimen- sion in the ionosphere during storms indicates a phase transition from higher values during the quiet periods to lower values during storm periods, which may be due to the modification of the ionosphere by the influx of high-intensity solar wind during the storm period (Unnikrishnan et al., 2006a, b; Unnikrishnan, 2010; Unnikrishnan and Ravindran, 2010).

The surrogate data test shows a significance of difference greater than 2 for all the computed measures, which enables rejection of the null hypothesis that the ionospheric system can be represented with a linear model for all the data used from the stations. However, it was discovered that the lower significance of difference corresponds to the lower values of Lyapunov exponents during storms and extremely disturbed periods (see Table 2a and $\mathrm{b}$ ). This may be due to the rise in stochasticity during the storm period as a result of a drop in values of computed quantities like Lyapunov exponents. Our ability to reject the null hypothesis for all stations however shows the presence of determinism and confirms that the underlying dynamics of the ionosphere is mostly nonlinear. This further validates the presence of chaos since the surrogate data test for non-linearity shows that our detrended TEC is not a Gaussian (linear) stochastic signal (Unnikrishnan, 2010). However, for some of the data sets, the saturation of the average mutual information observed at delay close to unity (as seen in Fig. 3) and the saturation Lyapunov exponent in the first time step (Fig. 6) indicate that there could be a high influence of stochasticity in the system.

The Tsallis entropy was able to show the deterministic behaviour of the ionosphere considering its response during storm periods compared to other relatively quiet periods, as the rapid drop in values of Tsallis entropy during storms shows that there is a transition from higher complexity during quiet periods to lower complexity during storms; this response in the values of the Tsallis entropy is similar to the response of Lyapunov exponent values during storms. This reaction to storms shown by the values of the Tsallis entropy computed for TEC was also described by the reaction of the Tsallis entropy computed for $D_{\text {st }}$ during storm periods (Balasis et al., 2008, 2009). A closer observation of the day-to-day variability within a month shows that the values were much lower for storm periods compared to the nearest relative quiet period. For example, the storm that occurred on 25 October resulted in lower values of Lyapunov exponents and the Tsallis entropy compared to relatively quiet days close to it. The reaction to storms may be due to the influence of a stochastic driver like strong solar wind flowing into the system as a result of solar flare or coronal mass ejections (CMEs) that produce the geomagnetic storms. Although there is always an influence of corpuscular radiation in the form of solar wind flowing from the Sun into the ionosphere, the influence is usually low for days without storms compared to days with geomagnetic storms as a result of solar flares, CMEs, etc. (Unnikrishnan et al., 2006a, b; Unnikrishnan, 2010; Ogunsua et al., 2014). 
The presence of chaos and high variations in the dynamical complexity, even in quiet periods in the ionosphere, may be due to the internal dynamics and inherent irregularities of the ionosphere which exhibit non-linear properties. However, this inherent dynamics may be complicated by external factors like geomagnetic storms. This may be the main reason for the drop in the values of Lyapunov exponents and Tsallis entropy during storms. According to Unnikrishnan et al. (2006a, b), geomagnetic storms are extreme forms of space weather, during which external driving forces, mainly due to solar wind, subsequent plasmasphere-ionosphere coupling, and related disturbed electric field and wind patterns, will develop. This in turn creates many active degrees of freedom with various levels of coupling among them, which alters and modifies the quiet time states of the ionosphere during a storm period. This new situation developed by a storm may modify the stability/instability conditions of the ionosphere, due to the superposition of various active degrees of freedom.

The observation from the day-to-day variability of the Lyapunov exponent and Tsallis entropy also shows irregular patterns for all stations. These irregular variations might be due to the same factors mentioned before (i.e. internal irregularities due to so many factors described and also due to variation in the influx of the external stochastic drivers). The day-to-day variability for the entire year shows a "wavelike" pattern with the values dropping to lower values during the equinox months, especially during the March-April equinox. This can be seen as a form of semiannual variation, possibly resulting from the higher energy inputs during equinoxes. This is because solar wind is maximized at the equinoxes, which might result in higher energy input that will eventually suppress the internal dynamics to give lower values of chaoticity. The modification of the ionosphere as a result of the higher energy input resulting from the maximized influx of solar wind has been reported as being responsible for the lower values of chaoticity when averagecompared to the days of the year with lower solar wind inputs as reported by Unnikrishnan et al. $(2006,2010)$ and Ogunsua et al. (2014). The semiannual pattern has been found to be similar for different stations, as seen in Figs. 7 and 12 and Figs. 9 and 13 for Lyapunov exponents and Tsallis entropy respectively. Figures 9 and 13 show the smoothed curves for Lyapunov exponents and Tsallis entropy respectively, with the drop in values at equinoxes showing more clearly. The phase transition in chaoticity and dynamical complexity is also responsible for the wavelike variations, with values of Lyapunov exponents and Tsallis entropy dropping during the equinoxial months, and this may be due to the influence of the daily influx of the solar wind having higher values during equinoxes due to the proximity of the Earth to the Sun during this period compared to the solstice months.

The wavelike pattern observed has been described as a result of the self-organized critical (SOC) phenomenon, a phenomenon which has been found to exist in both the mag- netosphere and the ionosphere or the space plasma system in general, due to coupling between the two systems, since the magnetosphere couples the ionosphere tightly to the solar wind (Lui, 2002). Many literatures have shown the existence of chaos in the SOC in the magnetosphere (Chang et al., 1992, 1998, 1999; Consolini et al., 1996; Chapman et al., 1998; Freeman and Watkins, 2002; Koselov and Koselova, 2001; Uritsky et al., 2003; Chang et al., 1992). The existence of SOC in the space plasma system involving both the ionosphere the the magnetosphere was described by Lui (2002) and Chang et al. (2002, 2004).

The variation along the latitude also shows the inconsistency and complexity of the ionospheric processes. This is the reason why, for the same day of the month, the values of Lyapunov exponents vary from one station to another. Lyapunov exponents however appear to respond better to changes in solar activities compared to Tsallis entropy, with more distinct results. This may be due to the fact that Tsallis entropy is not only a measure of complexity, but also that a measure of disorderliness in a system might not be as perfect in describing chaos as Lyapunov exponents. Kalogeropoulos (2012, 2013) and Baranger et al. (2002) observed that Tsallis entropy has a relationship that is not totally linear in all cases at different levels of chaos with Lyapunov exponents as a measure of chaos.

There are also many variations in the internal dynamics of the ionosphere that could lead to changes in chaotic behaviour. The variations of Lyapunov exponents during quiet days might be as a result of different variations in the intrinsic dynamics of the ionosphere. Differences in variation pattern at different stations for the same quiet day might also be due to the same reason. It can be affirmed that the ionosphere is a complex system that varies with a short latitudinal or longitudinal interval such that even stations with one or two degrees of latitudinal differences might record different values on the same day for both quiet and disturbed periods, and that the same might also occur for storm periods. This is illustrated by the different pattern of variation of TEC recorded from different stations within such a close range as used in this study.

These latitudinal variations in the values of Lyapunov exponents and Tsallis entropy can be further described by the behaviour of the TEC because there can be a more sporadic rate of change in TEC, as seen in the time series plots as a result of irregularities in the internal dynamics of the ionosphere, which might be as a result of plasma bubbles. Irregularities develop in the evening hours at $F$ region altitudes of the magnetic Equator, in the form of depletions, frequently referred to as bubbles. The edges of these depletions are very sharp, resulting in large time rates of TEC in the equatorial ionosphere, even during magnetically quiet conditions. The large gradient of the equatorial ionization persists in the local post-sunset hours till about 21:00 LT (DasGupta et al., 2007; Unnikrishnan and Ravindran, 2010). The TEC data for one station might experience an extremely sharp rate of change in 
TEC that may be due to some plasma bubbles in that region, while the TEC from the other station stays normal. These variations in the various internal dynamics like plasma bubbles leading to scintillation can cause variations in the dynamical response of the TEC. Hence, the irregular variation in the values of the Lyapunov exponent and Tsallis entropy, even in quiet periods for two relatively close stations, may be due to these irregularities. This might also be responsible for the quiet days in the same station that have lower values of Lyapunov exponents compared to higher values recorded for disturbed days without the external influence of storms.

The variations of these chaos and dynamical complexity parameters might also be a result of the anomalous TEC enhancements that might occur at night (Balan and Rao, 1987; Balan et al., 1991). These effects can also be seen more clearly in the Tsallis entropy values for the five-period window for quiet days of January 2011, because the nighttime value is higher and also shows a much higher series of fluctuations during this period compared to other periods. As mentioned in Unnikrishnan and Ravindran (2010), the irregular changes in the dynamical characteristics of TEC from the results of Lyapunov exponents and Tsallis entropy also may be due to the collisional Raleigh-Taylor instability, which may give rise to a few large irregularities in $\mathrm{L}$ band measurements (Rama Rao et al., 2006; Sripathi et al., 2008); all these can be seen as internal factors responsible for variations in the dynamical response of TEC as recorded from the values of the Lyapunov exponents and Tsallis entropy completed for days without storm, which might be quiet or disturbed according to classification and also could account for higher values of these qualifiers during disturbed days compared to quiet days. During storms, however, the values were much lower.

Earlier, we (Ogunsua et al., 2014) showed the appearance and variation of chaoticity quiet and disturbed day classification by the international most quiet day (IQD) and internal most disturbed day (IDD) classifications, as compared to quiet and storm periods used by Unnikrishnan $(2006,2010)$. We were able establish that a relatively quiet day may be less chaotic compared to a relatively disturbed day, unlike the result presented by Unnikrishnan $(2006,2010)$ for quiet and storm periods. Also, the combined use of both Lyapunov exponents and Tsallis entropy for the first time was found to have a high correlation mostly above $80 \%$, which has stimulated the interest in further research using the two diagnoses for the study of ionospheric dynamics.

This work on the other hand presents the results for day-today variation and has revealed a seasonal trend for both Lyapunov exponents and Tsallis entropy, which appear wavelike in form, with troughs during the two equinoxes. This was established for different stations used in this research work. The results show the appearance of seasonal trends in spite of the sporadic daily variation resulting from various changes in the internal dynamics. The seasonal trend has provided more possible evidence of higher energy input during equinoxes, since it reveals the effect of the annual energy input to the ionosphere. The day-to-day response of these parameters has also revealed the variations in the underlying dynamics of the system.

As a similarity between the present work and Ogunsua et al. (2014), the relationship between Lyapunov exponents and Tsallis entropy can also be seen from this work, as the two quantifiers exhibit similarities in their response to the dynamical behaviour of the ionosphere, with phase transition at the same periods of time for all stations. A further investigation of this relationship shows that all the daily values of Tsallis entropy correlate positively with the values of Lyapunov exponents, at values between 0.78 and 0.83 .

The ability of these quantifiers to clearly reveal the ionospheric dynamical response to solar activities and changes in its internal dynamics due to other factors is a valid proof of the authenticity of the use of these chaotic and dynamical measures as indices for ionospheric studies.

\section{Conclusions}

The chaotic behaviour and dynamical complexity of the low-latitude ionosphere over some parts of Nigeria were investigated using TEC time series measured simultaneously at five different stations, namely, Birnin Kebbi (geographic coordinates $12^{\circ} 32^{\prime} \mathrm{N}, 4^{\circ} 12^{\prime} \mathrm{E}$; dip latitude $0.62^{\circ} \mathrm{N}$ ), Torro (geographic coordinates $10^{\circ} 03^{\prime} \mathrm{N}, 9^{\circ} 04^{\prime} \mathrm{E}$; dip latitude $-0.82^{\circ} \mathrm{N}$ ), Enugu (geographic coordinates $6^{\circ} 26^{\prime} \mathrm{N}$, $7^{\circ} 30^{\prime} \mathrm{E}$; dip latitude $-3.21^{\circ} \mathrm{N}$ ), Lagos (geographic coordinates $6^{\circ} 27^{\prime} \mathrm{N}, 3^{\circ} 23^{\prime} \mathrm{E}$; dip latitude $-3.07^{\circ} \mathrm{N}$ ) and Yola (geographic coordinates $9^{\circ} 12^{\prime} \mathrm{N}, 12^{\circ} 30^{\prime} \mathrm{E}$; dip latitude $-1.39^{\circ} \mathrm{N}$ ) within the low-latitude region. The detrended TEC time series data obtained from the GPS data measurement were analysed using different chaoticity and dynamical complexity parameters.

The evidence of the presence of chaos in all the time series data was obtained for all the data used, as indicated by the positive Lyapunov exponent. The results of Tsallis entropy show the variations in the dynamical complexity of the ionosphere, which may be due to geomagnetic storms and other phenomena like changes in the internal irregularities of the ionosphere. The response of the Tsallis entropy to various changes in the ionosphere also shows the deterministic nature of the system. The results of the Tsallis entropy show a lot of similarities to that of the Lyapunov exponents between 0.78 and 0.81 , with both results showing a phase transition from higher values in the solstices to lower values during the equinoxial months. The values of Lyapunov exponents were found to be lower for the days of the months in which a storm was recorded relative to the nearest relatively quiet days, which agrees with previous works by other investigators. A similar pattern of results was obtained for the computed values of Tsallis entropy. The random variations in the values of chaoticity in the detrended TEC describing 
the internal dynamics of the ionosphere, as seen in the result obtained from both Lyapunov exponents and Tsallis entropy, depict the ionosphere as a system with a continuously changing internal dynamics, which shows that the ionosphere is not totally deterministic, but also has some elements of stochasticity influencing its dynamical behaviour.

The phase transition in the systems of the ionosphere resulting in the lower values of the chaoticity and dynamical complexity quantifiers during the geomagnetic storms and the equinoxial months is evidence that the ionosphere can be greatly modified by stochastic drivers like solar wind and other incoming particle systems. The drop in values during equinoxes can be seen as a form of semiannual variation, a phenomenon peculiar to the low-latitude regions.

Although the knowledge of being able to characterize the ionospheric behaviour using the two major quantifiers shows their ability to measure the level of determinism when used together, the relationship between these two quantifiers calls for more research, in the use of these qualifiers, to enable proper description and characterization of the state of the ionosphere. The response of both Tsallis entropy and Lyapunov exponents to changes in the ionosphere shows that the two quantifiers can be used as indices to describe the processes/dynamics of the ionosphere.

Acknowledgements. The authors appreciate the editorial team and the referees for their contributions which have led to the final shape of this paper. The GPS data used for this research were obtained from the public archives of the Office of the Surveyor General of the Federation (OSGoF) of the Federal Government of Nigeria, which is the mapping agency of Nigeria.

Edited by: A. S. Sharma

Reviewed by: four anonymous referees

\section{References}

Abdu, M. A.: Major Phenomena of the equatorial ionosphere thermosphere system under disturbed conditions, J. Atmos. Solten Phys., 59, 1505-1519, 1997.

Anastasiadis, A., Costa, L., Gonzáles, C., Honey, C., Széliga, M., and Terhesiu, D.: Measures of Structural Complexity in Networks, Complex Systems Summer School 2005, Santa Fe, 2005.

Baillie, R. and Chung, S.: Modeling and forecasting from trend stationary long memory models, with applications in climatology, Int. J. Forecast., 18, 215-226, 2002.

Balan, N. and Rao, P. B.: Latitudinal variations of nighttime enhancements in total electron content, J. Geophys. Res., 92, 34363440, 1987.

Balan, N., Bailey, G. J., and Balachandia, N. R.: Solar and Magnetic effects on the latitudinal variations of nighttime TEC enhancement, Ann. Geophys., 9, 60-69, 1991, http://www.ann-geophys.net/9/60/1991/.

Balasis, G. and Mandea, M.: Can electromagnetic disturbances related to the recent great earthquakes be detected by satellite magnetometers?, Tectonophysis, 431, 173-195, doi:10.1016/j.tecto.2006.05.038, 2007.

Balasis, G., Daglis, I. A., Papadimitrou, C., Kalimeri, M., Anastasiadis, A., and Eftaxias, K.: Dynamical complexity in $D_{\text {st }}$ time series using non-extensive Tsallis entropy, Geophs. Res. Lett., 35, L14102, doi:10.1029/2008GL034743, 2008.

Balasis, G., Daglis, I. A., Papadimitrou, C., Kalimeri, M., Anastasiadis, A., and Eftaxias, K.: Investigating Dynamical complexity in the magnetosphere using various entropy measures, J. Geopys. Res., 114, A00D06, doi:10.1029/2008JA014035, 2009.

Baranger, M., Latora, V., and Rapisarda, A.: Time evolution of thermodynamic entropy for conservative and dissipative chaotic maps, Chaos Soliton Fract., 12, 471-478, 2002.

Bhattacharyya, A.: Chaotic behavior of ionosphere turbulence from scintillation measurements, J. Geophys. Res., 17, 733-738, 1990.

Bhattacharyya, A. and Pandit, J.: Seasonal variationof spread-F occurrence probability at low latitude and its relation with sunspot number, Int. J. Elect. Commun. Technol., 5, 40-43, 2014.

Bloomfeld, P.: Trends in global Temperature, Climate Change, 21, 1-16, 1992.

Bloomfield, P. and Nychka, D.: Climate spectra and detecting climate change, Climate Change, 21, 275-287, 1992.

Boon, J. and Tssallis, C. (Eds.): Nonexistensive statistical mechanics: New trends, new perspectives, Europhys. News, 36, 185231, 2005.

Burgula, L. F., Vixas, A. F., and Wang, C.: Tsallis distribution of magnetic field strength variations in the heliosphere: 5 to $90 \mathrm{AU}$, J. Geophys. Res., 112, A07206, doi:10.1029/2006JA012213, 2007.

Chang, T.: Low-Dimensional Behavior and Symmetry-Breaking of Stochastic-Systems Near Criticality-Can These Effects Be Observed in Space and in the Laboratory, IEEE T. Plasma Sci., 2, 691-694, 1992.

Chang, T.: Sporadic localized reconnection and multiscale intermittent turbulence in the magnetotail, in: AGU Monograph No. 104 Geospace Mass and Energy Flow, edited by: Horwitz, J. L., Gallagher, D. L., and Peterson, W. K., American Geophysical Union, Washington, D.C., p. 193, 1998.

Chang, T.: Self-organized criticality, multi-fractal spectra, sporadic localized reconnections and intermittent turbulence in the magnetotail, Phys. Plasmas, 6, 4137-4145, 1999.

Chapman, S. C., Watkins, N. W., Dendy, R. O., Helander, P., and Rowlands, G.: A simple avalanche model as an analogue for magnetospheric activity, Geophys. Res. Lett., 25, 2397-2400, 1998.

Coco, I., Consolini, G., Amata, E., Marcucci, M. F., and Ambrosino, D.: Dynamical changes of the polar cap potential structure: an information theory approach, Nonlin. Processes Geophys., 18, 697-707, doi:10.5194/npg-18-697-2011, 2011.

Consolini, G., Marcucci, M. F., and Candidi, M.: Multifractal structure of auroral electrojet index data, Phys. Rev. Lett., 76, 40824085, 1996.

Coraddu, M., Lissia, M., and Tonelli, R.: Statistical descriptions of nonlinear systems at the onset of chaos, arXiv:condmat/0511736v1, 30 November 2005.

Cosolini, G. and Chang, T.: Magnetic field topology and criticality in geotail dynamics relevance to substorm phenomena, Space Sci. Rev., 95, 309-321, 2001. 
DasGupta, A. and Das, A.: Ionospheric total electron content (TEC) studies with GPS in the equatorial region, India J. Radio Space Phys., 36, 278-292, 2007.

Fraser, A. M. and Swinney, H. L.: independent coordinates for storage attractors from mutual information, Phys. Rev. A, 33, 11341141, 1986.

Freeman, M. P. and Watkins, N. W.: The heavens in a pile of sand, Science, 298, 979-980, 2002.

Fuller-Rowell, T. J., Codrescu, M. V., Moffett, R. J., and Quegan, S.: Response of the magnetosphere and ionosphere to geomagnetic storms, J. Geophys. Res., 99, 3893-3914, 1994.

Grassberger, P. and Procaccia, I.: Characterization of strange attractors, Phys. Rev. Lett., 50, 346-349, 1983a.

Grassberger, P. and Procaccia, I.: Measuring the strangeness of strange attractors, Physica D, 9, 189-208, 1983b.

Hegger, R., Kantz, H., and Shreber, T.: Practical implementation of nonlinear time series method. The Tisean package, Chaos, 9, 413-430, 1994.

Kalogeropoulos, N.: Weak chaos from Tsallis entropy, Qscience Connect, 12, doi:10.5339/connect.2012.12, 2012.

Kalogeropoulos, N.: Vanishing largest Lyapunov exponent and Tsallis entropy, Qscience Connect, 26, doi:10.5339/connect.2013.26, 2013.

Kantz, H. and Shreber, T.: Nonlinear time series analysis, 2nd Edn., Cambridge University Press, 69-70, 2003.

Kazimirovsky, E. S. and Vergasova, G. V.: Mesospheric, Lower Thermospheric Dynamics and External Forcing Effects: A Review, Indian J. Radio Space Phys., 38, 7-36, 2009.

Kazimirovsky, E. S., Kokourov, V. D., and Vergasova, G. V.: Dynamical Climatology of the Upper Mesosphere, Lower Thermosphere and Ionosphere, Surv. Geophys., 27, 211-255, 2006.

Kennel, M. B., Brown, R., and Abarbanel, H. D. I.: Determining minimum embedding dimension using a geometrical construction, Phys. Rev. A, 45, 3403-3411, 1992.

Kim, S., Koh, K., Boyd, S., and Gorivesky, D.: $L_{1}$ Trend filtering, SIAM Review, 51, 339-360, 2009.

Klobuchar, J.: Design and characteristics of the GPS ionospheric time-delay algorithm for single frequency users, in: Proceedings of PLANS' 86 - Position Location and Navigation Symposium, 4-7 November 1986, Las Vegas, Nevada, 280-286, 1986.

Kozelov, B. V. and Kozelova, T. V.: Sandpile model analogy of the magnetosphere-ionosphere substorm activity, Proc. Interball Meeting, Warsaw, Poland, 2001.

Kumar, K. S., Kumar, C. V. A., George, B., Renuka, G., and Venugopal, C.: Analysis of the fluctuations of the total electron content, measured at Goose Bay using tools of nonlinear methods, J. Geophys. Res., 10, A02308, doi:10.1016/j.tecto.2006.05.038, 2007.

Lui, A. T. Y.: Evaluation on the analogy between the dynamic magnetosphere and a forced and/or self-organized critical system, Nonlin. Processes Geophys., 9, 399-407, doi:10.5194/npg9-399-2002, 2002.

Mukherjee, S., Shivalika, S., Purohit, P. K., and Gwal, A. K.: Study of GPS ionospheric scintillations over equatorial anomaly station Bhopal, Int. J. Adv.n Earth Sci., 1, 39-48, 2002.

Ogunsua, B. O., Laoye, J. A., Fuwape, I. A., and Rabiu, A. B.: The comparative study of chaoticity and dynamical complexity of the low-latitude ionosphere, over Nigeria, during quiet and disturbed days, Nonlin. Processes Geophys., 21, 127-142, doi:10.5194/npg-21-127-2014, 2014.

Pavlos, G. P., Kyriakov, G. A., Rigas, A. G., Liatsis, P. I., Trochoulos, P. C., and Tsonis, A. A.: Evidence for strange attractor structures in space plasma, Ann. Geophys., 10, 309-315, 1992, http://www.ann-geophys.net/10/309/1992/.

Perreault, P. and Akasofu, S.-I.: A study of geomagnetic storms, Geophys. J. R. Astron. Soc., 54, 547-573, 1978.

Rabiu, A. B., Mamukuyomi, A. I., and Joshua, E. O.: Variability of equatorial ionosphere inferred from geomagnetic field measurement, Bull. Astro Soc. India, 35, 607-615, 2007.

Rama Rao, P. V. S., Gopi Krishna, S., Niranjan, K., and Prasad, D. S. V. V. D.: Temporal and spatial variations in TEC using simultaneous measurements from the Indian GPS network of receivers during the low solar activity period of 2004-2005, Ann. Geophys., 24, 3279-3292, doi:10.5194/angeo-24-3279-2006, 2006.

Reddy, D. S., Reddy, N. G., Radhadevi, P. V., Saibaba, J., and Varadan, G.: Peakwise smoothing of data models using wavelets, World Academy of Science, Engineering and Technology, Turkey, 2010.

Remya, R. and Unnikrishnan, K.: Chaotic Behaviour of interplanetary magnetic field under various geomagnetic conditions, J. Atmos. Sol.-Terr. Phys. 72, 662-675, 2010.

Rosenstein, M. T., Collins, J. J., and DeLuca, C. J.: A practical method for calculation Largest Lyapunov Exponents from small Data sets, Physca D, 65, 117-134, 1993.

Saito, A., Fukao, S., and Mayazaki, S.: High resolution mapping of TEC perturbations with the GSI GPS network over Japan, Geophys. Res. Lett., 25, 3079-3082, 1998.

Savitzky, A. and Golay, M. J. E.: Smoothing and differentiation by simplified least square procedures, Anal. Chem., 36, 1627-1639, 1964.

Shan, H., Hansen, P., Goertz, C. K., and Smith, K. A.: Chaotic appearance of the ae index, J. Geophys. Res., 18, 147-150, 1991.

Sindelarova, T., Buresova, D., and Chum, J.: Observations of acoustic-gravity waves in the ionosphere generated by severe tropospheric weather. Studia Geophysica et Geodaetica, 53, 403418, doi:10.1007/s11200-009-0028-4, 2009.

Strogatz, S. H.: Nonlinear Dynamics and Chaos, Addison-Wesley Publishing Company, Reading, Massachusetts, 412-415, 1994.

Tsallis, C.: Possible generalization of Boltzmann-Gibbs statistics, J. Stat. Phys., 52, 487-497, 1988.

Tsallis, C.: Generalised entropy-based criterion for consistent testing, Phys. Rev. E., 58, 1442-1445, 1998.

Tsallis, C.: Nonextensive statistics: theoretical, experimental and computational evidences and connections, Braz. J. Phys., 29, 135,1999 .

Unnikrishnan, K.: Comparison of chaotic aspects of magnetosphere under various physical conditions using AE index time series, Ann. Geophys., 26, 941-953, doi:10.5194/angeo-26-941-2008, 2008.

Unnikrishnan, K.: A comparative study on chaoticity of equatorial/low latitude ionosphere over Indian subcontinent during geomagnetically quiet and disturbed periods, Nonlin. Processes Geophys., 17, 765-776, doi:10.5194/npg-17-765-2010, 2010.

Unnikrishnan, K. and Ravindran, S.: A study on chaotic behavior of equatorial/low latitude ionosphere over indian subcontinent, using Gps - TEC time series, J. Atmos. Sol.-Ter. Phys., 72, 10801089, 2010. 
Unnikrishnan, K., Saito, A., and Fukao, S.: Differences in magnetic storm and quiet ionospheric deterministic chaotic behavior: GPS TEC Analyses, J. Geophys. Res., 111, A06304, doi:10.1029/2005JA011311, 2006a.

Unnikrishnan, K., Saito, A., and Fukao, S.: Differences in day and night time ionosphere determine chaotic behavior: GPS TEC Analyses, J. Geophys. Res., 111, A07310, doi:10.1029/2005JA011313, 2006b.

Uritsky, V. M., Klimas, A. J., and Vassiliadis, D.: Evaluation of spreading critical exponents from the spatiotemporal evolution of emission regions in the nighttime aurora, Geophys. Res. Lett., 30, 1813, doi:10.1029/2002GL016556, 2003.

Vassiliadis, D .V., Sharma, A. S., Eastman, T. E., and Papadopoulos, K.: Low-dimensionless chaos in magnetospheric activity from AE time series, Geophys. Res. Lett., 17, 1841-1844, 1990.
Vyas, G. D. and Chandra, H.: VHF scintillations and spread-F in the anomaly crest region, Indian J. Radio .Space Phys., 23, 15-164, 1994.

Vyas, R. M. and Dayanandan, B.: Night time VHF ionospheric scintillation characteristics near crest of Appleton anomaly stations, Udaipur $26\left(^{\circ} \mathrm{N}, 73^{\circ} \mathrm{E}\right)$, Indian J. Radio Space Phys., 40, $191-$ 202, 2011.

Wernik, A. W. and Yeh, K. C.: Chaotic behavior of ionospheric scintillation medelling and observations, Radio Sci., 29, 135-139, 1994.

Wolf, A., Swift, J. B., Swinney, H. L., and Vastano, J. A.: Determining Lyapunov exponents from a time series, Physica D, 16, 285-317, doi:10.1016/0167-2789(85)90011-9, 1985. 